\title{
Peculiarities of problem solving at studying well drilling with the use of linear differential equations with constant coefficients
}

\author{
Igtisamova Galia Rashitovna \\ Ufa State Petroleum Technological University, \\ Branch of the University in the City of Oktyabrsky, Russian \\ Federation \\ E-mail: igtisamova_galiy@ mail.ru
}

\begin{abstract}
- this article introduces a variation of the parameter method for solving homogeneous linear ordinary differential equations of the second order with constant coefficients, which are most often encountered in the oil and gas engineering when studying the drilling, production and pipeline transport of oil and gas. In addition, we derive a general method of solution for solving homogeneous linear ordinary differential equations of the second order with constant coefficients.
\end{abstract}

Keywords - differential equation; the equation of oscillations; linear combination; oil and gas industry, drill pipes.

\section{INTRODUCTION}

The article deals with differential equations, used in oil engineering for studying well drilling processes, oil extractions, and pipe line oil and gas transport [1,2]. Partial differential equations are often called equations of mathematical physics. For example, describing reflex movements of the layer due to mechanical action on it (for example, by a drill) Laplace equation is used. A layer is considered as a membrane [3, 4]. Describing rod string or stem movement we use a wave equation [5-8].

Studying these processes it is better to use mathematical method of solving differential equations $[4,9]$. A differential equation is a function connecting an unknown function of one or several variables its derivatives with respect to one or more unknown variables [10]. If an unknown function depends only on one independent variable, this differential equation is an ordinary differential equation. The order of the ordinary differential equation is equal to the order of the higher derivative, which appears in the equation [11]. A differential equation with unknown function is linear if it is linear to unknown function and its derivatives. Difference equation $a y^{(2)}+b y^{(1)}+c y=f(x)$ is linear, ordinary differential equation with constant coefficients, where $\mathrm{a}, \mathrm{b}$ and $\mathrm{c}$ are invariables, a is a non-zero scalar; $f(x)$ is $x$ depending function. Linear differential equation with invariable coefficients is as follows: $a y^{(2)}+b y^{(1)}+c y=f(x)$, $a y^{(2)}+b y^{(1)}+c y=0$. At first we solve linear ordinary differential equation of the second order with constant coefficients $a y^{(2)}+b y^{(1)}+c y=f(x)$. Polynom $a p^{2}+$ $b p+c$ is a characteristics of $a y^{(2)}+b y^{(1)}+c y=0$. Function $y=\exp \int m d x$ is a solution for $a y^{(2)}+b y^{(1)}+$

\author{
Nosirov Donier Shukurilloevich \\ Ufa State Petroleum Technological University, \\ Branch of the University in the City of Oktyabrsky, Russian \\ Federation \\ E-mail: igtisamova_galiy@mail.ru
}

$c y=0$ only when $m$ is a root of this polynom $a p^{2}+b p+c$ $[5,12,13]$. The equation $a y^{(2)}+b y^{(1)}+c y=0$ is solved through the function $y=\exp \int m d x$ any time when $m$ is a number that $a m^{2}+b m+c=0$. We characterize the equation $a m^{2}+b m+c=0$ to solve $a y^{(2)}+b y^{(1)}+c y=0$.

It is evident that a standard equation $a m^{2}+b m+c=0$ is a square equation in $\mathrm{m}$. We have three cases of a standard equation solution $a m^{2}+b m+c=0$ and $y^{(2)}+b y^{(1)}+$ $c y=0$. Authors of the books on differential equations used this method to solve linear ordinary differential equations of the second order with constant coefficients. Let's apply Moiver's theorem to find common solution $a y^{(2)}+b y^{(1)}+$ $c y=0$ with $b^{2}-4 a c<0$ [14].

This article introduces a variant of a parameter method to find common solution of a homogenous linear ordinary differential equation of the second order with constant coefficients. If we use Moiver's theorem to find common solutions of a homogenous linear ordinary differential equation of the second order with constant coefficients and if we change a scalar $m$ into function $u(x)$, supposing that $y=\exp \int m d x$ is a solution of a homogenous linear ordinary differential equation of the second order with constant coefficients then $y=\exp \int m d x$ is a parameters method variation to solve linear ordinary differential equation of the second order with constant coefficients, where $u(x)$ is $x$ function. Finally, we determine $u(x)$ from $y=\exp \int m d x$, taking it as a solution of the homogenous linear ordinary differential equation of the second order with constant variables.

\section{THE PURPOSE OF THE RESEARCH}

To determine the function $u(x), y=\exp \int m d x$, which is a solution of the ordinary differential equation of the second type with constant coefficients.

\section{LINEAR DIFFERENTIAL EQUATIONS OF THE FIRST ORDER}

Linear differential equation of the first order with unknown dependent variable and $y$ and independent $x$ is determined by formula $a_{0}(x) y+a_{1}(x) y^{(1)}=g(x)$. 
According to mathematical transformations we receive the second solution $y_{2}(x)=u(x) y_{1}(x)$ in the equation (3) $y_{2}(x)=y_{1}(x) \int v d x$, where $\quad v=\frac{1}{\mu(x)} \quad$ and $\mu(x)=$ $y_{1}^{2} \exp \left(\int\left(\frac{b}{a}\right) d x\right)$.

From theorem 1 we note that $y=c_{1} y_{1}+c_{2} y_{2}$ is a common solution of the equation (3), where $y_{1}$ and $y_{2}$ are linearly independent solutions of the equation from (3).

Common solution of the homogenous linear ordinary differential equations of the second type with constant coefficients

Basing on the common solution of a linear ordinary (1)

$a_{0}(x) y+a_{1}(x) y^{1}+a_{2}(x) y^{(2)}=g(x)$.

Main solution set: variety of functions $y_{1}(x), y_{2}(x), \ldots y_{n}(x)$ is linearly dependent on interval $\mathrm{I}$, if there are constants $c_{1}, c_{2}, \ldots c_{n}$, also they are called linearly dependent on interval $\mathrm{I}$, if there are constants $c_{1} y_{1}(x)+$ $c_{2} y_{2}(x)+\cdots+c_{n} y_{n}(x)=0$ for each $x$ in the interval. If the set of functions is linearly independent of the interval then it is then it is called linearly independent. Any variety $y_{1}(x), y_{2}(x), \ldots y_{n}(x)$ from $n$ of linearly independent solutions of a homogenous linear $n$-differential equation in the interval I is called a main solution set on the section [12].

Common solution of linear differential equations of the second type: homogenous differential equation, connected with inhomogeneous linear differential equation $n$-th order, is: $g(x)$

$a_{0}(x) y+a_{1}(x) y^{(1)}+\cdots+a_{n-1}(x) y^{(n-1)}+a_{n}(x) y^{(n)}=$

and

$a_{0}(x) y+a_{1}(x) y^{(1)}+\cdots+a_{n-1}(x) y^{(n-1)}+a_{n}(x) y^{(n)}=$ 0 . Theorem 1. Let $y_{1}(x), y_{2}(x), \ldots y_{n}(x)$ are linearly independent solutions of the homogenous linear differential equation of the second type of the $n$-th order

(2)

$$
a_{0}(x) y+a_{1}(x) y^{(1)}+\cdots+a_{n-1}(x) y^{(n-1)}+a_{n}(x) y^{(n)}=0
$$

on the interval I. Then common solution of the equation (2) on the section I will be:

$y=c_{1} y_{1}+c_{2} y_{2}+\cdots+c_{n-1} y_{n-1}+c_{n} y_{n}$,

where $c_{i},(\mathrm{i}=1,2, \ldots, \mathrm{n}-1, \mathrm{n})$ are arbitrary constants [16]. Common solution of the equation (1) $y=c_{1} y_{1}+c_{2} y_{2}+y_{p}$. $y_{1}, y_{2}$ are linearly independent solutions of the associated homogenous equation (1), and $y_{p}$ is a specific solution of the equation (1). Here, $c_{i},(i=1,2)$ are arbitrary constants [15].

Constructing the second solution from the known solution: Let $a y^{(2)}+b y^{(1)}+c y=0$

is an homogenous linear ordinary differential equation of the second order with constant coefficients. Let $y_{1}$ a solution of the equation not equal to zero. (3). Then we have $c y_{1}+$ $b y_{1}^{(1)}+a y_{1}^{(2)}=0$.

Then we suppose that $y_{2}(x)=u(x) y_{1}(x)$ is another solution of the equation (3). To make the second solution of the equation (3) it follows that as $u=\int v d x$, then

$$
v=\frac{1}{\mu(x)} \text { and } \mu(x)=y_{1}^{2} \exp \left(\int\left(\frac{b}{a}\right) d x\right) .
$$

differential equation of the second order $a y^{(2)}+b y^{(1)}+c y=$ 0 with constant coefficients $a, b$ and $c y=c_{1} x \cdot \exp \alpha x+c_{2}$. exp $\alpha x$.

Let's suppose that $y=\exp \int u(x) d x$. It follows that $y^{(1)}=u y$ and $y^{(2)}=\left(u^{(1)}+u_{2}\right) y$.

As $y=\exp \int u(x) d x$ are solutions of the equations (3), than we have $a\left(u^{(1)}+u^{2}\right) y+b u y+c y=0$. When $y$ is not equal to naught, the function is as follows:

$a\left(u^{(1)}+u^{2}\right) y+b u+c=0$.

As a result

$y_{2}(x)=y_{1}(x) u=y_{1}(x)\left[\left(-\frac{1}{2 \beta}\right) \frac{\exp -\beta x}{(\exp \beta x+\exp -\beta x)}\right]=$ $\left(-\frac{1}{2 \beta}\right) \exp (\alpha-\beta) x$.

Hence, $y=c_{1} \exp (\alpha+\beta) x+\exp (\alpha-\beta) x$ is a common solution of the equation (3), as $y$ is a linear combination $y_{1}$ and $y_{2}$, if $4 a c-b^{2}<0, \alpha=\frac{-b}{2 a}$ и $\beta=\frac{\sqrt{b^{2}-4 a c}}{2 a}$.

Most authors of the differential equations used Moiver's theorem to find common solution $a y^{(2)}+b y^{(1)}+c y=0$ with $4 a c-b^{2}<0$ [14]. Here we used a variation of the parameters method to find common solution of a homogenous linear ordinary differential equation of the second order with constant coefficients. Besides we find common solution of a homogenous linear ordinary differential equation of the second order with constant coefficients without Moiver's theorem. Now let's study these equations from the point of view of oil engineering, precisely, well drilling. When lowering the drill tubes into the downhole, the "tube" is considered to be a "straight line" in the mathematical model. As the drill tubes diameter is less than the casing diameter, the tube oscillates during lowering, and these oscillations in oil engineering are described as "oscillation equation". Let's study these equations and solve them.

Let's study string vibration between two points. Let's take the following assumptions:

1) Tensile force extended along the string is so great that bending resistance can be neglected in comparison with the tensile force. The string is straight linear in rest; 
2) Let's consider flat vibrations, that is when all the string points are in one and the same fixed plane at any given moment of time.

Let's take this plane as the coordinate system $X O U$ (pic. $1)$. Let's denote the bend string equation at the moment $t$ $u=u(x, t)$. We will suppose this function to be twice continuously differentiable.

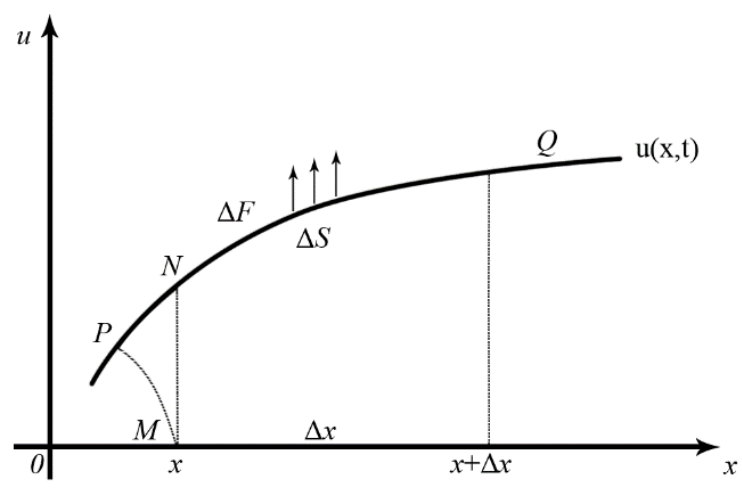

Fig. 1. Bend string equation graph at the moment

3) Let's consider only small vibrations, those when string declination from position of equilibrium is insignificant;

4) Let's suppose that string vibrations are cross axial, that is when each string point trajectory (for example, it takes position $M$ in the state of equilibrium) is not curvilinear (that corresponds reality), but straight linear, perpendicular axis $O x$. The real trajectory MP is substituted by segment $\mathrm{MN}$ (pic. 1). Taking this supposition point velocity projection $\mathrm{N}$ onto axis $O x$ is equal naught, and onto axis $O u$ equals $\frac{d u(x, t)}{d t}$. Accelleration projections $\mathrm{N}$ are equal naught and $\frac{d^{2} u}{d t^{2}}$

5) Let's suppose that external forces acting on a moving string are parallel axis $O u$. If $\Delta F$ is a projection onto axis $O u$ of the external forces, acting onto the string section $\Delta S$ (pic. 1 ), let's denote by $f(x, t)$ value $\frac{1}{\rho} \lim _{\Delta S \rightarrow 0} \frac{\Delta F}{\Delta S}$.

As $\Delta S$ and $\Delta x$ are equal

$$
f(x, t)=\frac{1}{\rho} \lim _{\Delta x \rightarrow 0} \frac{\Delta F}{\Delta S}=\frac{d F}{d m} .
$$

Then we see that $f(x, t)$ is the external force magnitude, rated for string unit mass. It is called force intensity.

Let's derive string vibration equation. Let $T$ be a string tension force vector. If accourding to an example from mechanics, we cut out a segment $N_{1} N_{2}$ from a string (pic. 2), than the influence of the discarded system parts on this segment will be equal to forces action $T_{1}(x, y)$ and $T_{2}(x, t)$, directed along the tangent to the arc $N_{1} N_{2}$, but in different directions.

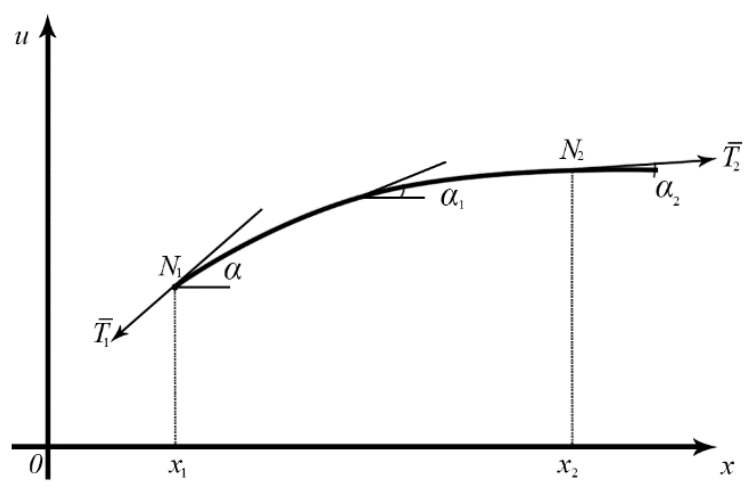

Fig. 2. Forces action $\mathrm{T}(\mathrm{x}, \mathrm{y})$ on the string

If $\alpha=\alpha(x, t)$ is a sharp angle between $\mathrm{X}$ axis and a tangent line to the string in the point with axis $x$ at the moment $t$, then the assumption of little vibrations is that value $\alpha^{3}$ may be neglected.

As $\sin \alpha=\alpha-\frac{\alpha^{3}}{31}+\cdots$, than we can consider $\sin \alpha \approx \alpha$, and it corresponds to reality as the trigonometric function $\sin \alpha$ at small values of the angle is equal to the angle itself $\cos \alpha \approx 1$. And finally, $\operatorname{tg} \alpha \approx \sin \alpha$. Where $\operatorname{tg} \alpha \approx \sin \alpha$.

Let's prove that with our assumptions tensile force $T$ can be considered constant, independent both of the point of its application and of timet. As according to the condition all string points move parallel axis $O u$ and external forces are parallel to this axis, then the sum of the tensile force projections on axis $O x$ is equal zero:

$-T_{1} \cos \alpha_{1}+T_{2} \cos \alpha_{2}=0$.

As $\cos \alpha \approx 1$ we conclude that $T_{1}=T_{2}$.

As it has been said above, forces $\mathrm{T}_{1}$ and $\mathrm{T}_{2}$ are tungsten to the string, their magnitude is constant and equal $T_{0}$. According to the formula (4) forces projections sum $\mathrm{T}_{1}$ and $\mathrm{T}_{2}$ onto $O x$ equals naught. Let's calculate the total of these forces projections onto the axis $\mathrm{Ou}$ :

$$
-T_{0} \sin \alpha_{1}+T_{0} \sin \alpha_{2}=T_{0}\left(\sin \alpha_{1}-\sin \alpha_{2}\right)=\frac{\partial u}{d x} .
$$

Relation (5) is written for the string segment, projected at interval $(x, x+d x)$ of $\mathrm{X}$ axis according to Lagrange theorem. The external forces resultant, applied to the segment $d x$, will approximately be equal to $f(x, t) d x$.

Now we have found all the forces, acting on the segment $d x$. Let's apply the second Newton's law according to which the product of mass by acceleration is equal to the total of all the acting forces (as the segment is small it can be taken as a point).

String segment mass is equal $\rho d x$, acceleration $\partial x^{2}$ :

$\rho d x \frac{\partial^{2} u}{\partial t^{2}}=T_{0} \frac{\partial^{2} u}{\partial x^{2}} d x+f(x, t) d x$,

or

$\frac{\partial^{2} u}{\partial t^{2}}=a^{2} \frac{\partial^{2} u}{\partial x^{2}}+\frac{1}{\rho} f(x, t)$, 
where $a^{2}=\frac{T_{0}}{\rho}$.

This equation is linear differential equation in the second order partial derivatives with constant coefficients and is called string vibration equation or one-dimensional wave equation. If $f(x, t)=0$, then the equation (6) is homogenous. Then it describes free vibrations of the string without action of the external forces.

\section{INITIAL AND BOUNDARY CONDITIONS FOR STRING VIBRATION EQUATION}

As it has been noted above, differential equations in second order partial derivatives have innumerable number of solutions depending on two arbitrary functions. To determine arbitrary functions, that is to find specific solutions it is necessary $\mathrm{t} o$ apply additional conditions onto the required function, $u(x, t)$. The same situation was while solving ordinary differential equations, when search of arbitrary constant from the complementary function was done according to the specified initial conditions.

In problems about string vibrations additional conditions may be of two types: initial and boundary. Initial conditions show, what the state of the string was at the moment of vibration. The initial position of the string points is given by the condition $\left.u(x, t)\right|_{t=0}=f(x)$, and the initial speed is $\left.\frac{\partial u}{\partial t}\right|_{t=0}=F(x)$, where $f(x)$ and $F(x)$ are specified functions.

Boundary conditions show what happens at the string ends during oscillation. In the simplest case, when the string ends are fixed, function $u(x, t)$ must be dependent on the conditions $u_{x=0}=0, u_{x}=0$.

Let's formulate the mathematical problem, which is driven from the study of free vibrations of the string fixed at both ends.

Let's solve a homogenous linear differential equation in partial derivatives with a constant coefficient $\frac{\partial^{2} u}{\partial t^{2}}=a^{2} \frac{\partial^{2} u}{\partial x^{2}}$.

At the initial conditions $u_{t=0}=0 ;\left.\quad \frac{\partial u}{\partial t}\right|_{t=0}=F(x)$,

And boundary conditions $u_{x=0}=0, u_{x}=0$, where $u_{1}(x)$ is stationary condition determined by the problem solution.

\section{DRILL STEM VIBRATIONS DURING LOWERING}

To determine dynamic strength in the drill stem with the account of longitudal tensile vibrations let's suppose, that during descending the stem is stopped by casing spiders (instantly), having at the time moment $t_{0}$ speed $v_{0}$ and acceleration $g$. Differential equation of the stem motion after stopping is: $\frac{\partial^{2} u}{\partial t^{2}}=a^{2} \frac{\partial^{2} u}{\partial x^{2}}+\boldsymbol{q}$.

Let's assume that after stopping the bottom end is influenced by flush fluid static pressure. Then boundary conditions will be $\left.u\right|_{x=0}=0,\left.\quad E \frac{\partial u}{\partial t}\right|_{x}=-y_{2}$, where $y_{2}$ is relative weight of the mud solution.

The initial conditions are as follows: $\left.u\right|_{x=0}=-\frac{y_{2} x}{E}, \frac{d u}{d t}=v_{0}$.
Let's express stem oscillation period during lowering from the formula (5):

$$
T=C \cos a u t+D \sin a u_{1} t .
$$

If we associate the vibrations with the stem lowering speed then it will be:

$$
v_{1}=\frac{a}{E}\left(\sigma_{T}-\left(y_{1}-y_{2}\right) t\right)
$$

\section{CONCLUSION}

Here we have determined indefinite function $u(x)$, supposing $y=\exp \int u(x) d x$ is a solution of the homogenous linear ordinary differential equation of the second order with constant coefficients. Moreover, we proved application of a variational parameter for solving homogenous linear ordinary differential equation of the second order with constant coefficients, which are widely used in oil engineering in solving complicated engineering problems.

\section{References}

[1] R.M. Shaidullina, A.F. Amirov, V.S. Muhametshin, and K.T. Tyncherov, "Designing economic socialization system in the educational process of technological university," European Journal of Contemporary Education, Vol. $6 \quad(1), \quad 2017, \quad$ pp. 149-158. DOI: 10.13187/ejced.2017.1.1494.

[2] K.T. Tyncherov, M.V. Selivanova Modular wavelet approach for reinforced plastic pipelines operation quality evaluation, International Conference on Industrial Engineering, Applications and Manufacturing (ICIEAM) (Saint Petersburg, 16-19 May 2017), 2017.

[3] L.J. Crane, Flow past a stretching plate, ZAMP, Vol. 21, pp. 645-647, 1970.

[4] G.Z. Dennis, A First Course in Differential Equations. Ricard Stratton, Los Angeles, United States of America, 2013.

[5] C.C. Lin, Note on a class of exact solutions in magneto-hydrodynamics, Arch. Rational Mech. Anal., Vol.1. pp. 391-395, 1958.

[6] K. F. Gabdrakhmanova, F. K. Usmanova, G. A. Khramov "Probabilistic and Statistical Approach as a Means of Predicting the Efficiency of Hydraulic Fracturing", Eastern-European Journal of Enterprise Technologies, Vol. 3, 8(81), pp. 30-36, 2016.

[7] G.R. Igtisamova N.O. Kovalev, Reliability evaluation for improved screw dies of coiled tubing unit injector, Tribology in Industry, Vol. 391, pp. 45-49, 2017.

[8] K.T. Tyncherov, V.Sh. Mukhametshin, and L.B. Khuzina, "Method to control and correct telemtry well information in the basis of residue number system," Journal of Fundamental and Applied Sciences, vol. 9, no. 2S, pp. 1370-1374, 2017. DOI: 10.4314/jfas.v9i2s.848.

[9] I. P. Uteshev, Some aspects of linear differential equations, RadioTechnical Notes, Vol. 55, pp. 41-47, 2015.

[10] G.Z. Dennis, A First Course in Differential Equations. Ricard Stratton, Los Angeles, United States of America, 2013.

[11] S. Yuksel (2014) Differential Equations for Engineering Science. Queen's University, Canada.

[12] R.B. Ogunrinde, J. Sunday, On Some Models Based on Second Order Differential Equations, Am J Sci Ind Res, Vol. 3, pp. 288-291, 2012.

[13] K. Tamada, Two-dimensional stagnation point flow impinging obliquely on a plane wall, J. Phys. Soc. Jap., Vol. 46, pp. 310-311, 1979.

[14] D. Gonze, W. Abou-Jaoude, Linear differential Equations, 2012.

[15] D. Arficho, Method for Solving Particular Solution of Linear Second Order Ordinary Differential Equations, J Appl Computat Math, Vol. 4, pp. 210, 2015.

[16] D. Levermore, Higher-Order Linear Ordinary Differential Equations I: Introduction and Homogeneous Equations, Department of Mathematics, University of Maryland, 2012. 